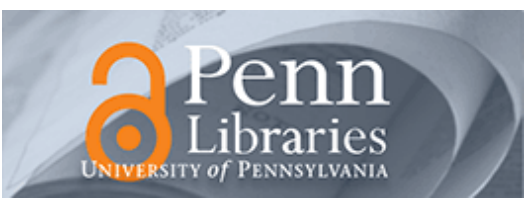

University of Pennsylvania

ScholarlyCommons

6-13-2009

\title{
Ambipolar transport in solution-deposited pentacene transistors enhanced by molecular engineering of device contacts
}

\author{
Sangameshwar Rao Saudari \\ University of Pennsylvania, saudari@seas.upenn.edu \\ Paul R. Frail \\ University of Pennsylvania \\ Cherie R. Kagan \\ University of Pennsylvania, kagan@seas.upenn.edu
}

Follow this and additional works at: https://repository.upenn.edu/ese_papers

Part of the Electrical and Computer Engineering Commons

\section{Recommended Citation}

Sangameshwar Rao Saudari, Paul R. Frail, and Cherie R. Kagan, "Ambipolar transport in solution-deposited pentacene transistors enhanced by molecular engineering of device contacts", . June 2009.

Suggested Citation:

Saudari, S.R., Frail, P.R., Kagan, C.R. (2009). "Ambipolar transport in solution-deposited pentacene transistors enhanced by molecular engineering of device contacts." Applied Physics Letters. 95, 023301.

(C) 2009 American Institute of Physics. This article may be downloaded for personal use only. Any other use requires prior permission of the author and the American Institute of Physics.

The following article appeared in Applied Physics Letters and may be found at http://dx.doi.org/10.1063/

1.3177007

This paper is posted at ScholarlyCommons. https://repository.upenn.edu/ese_papers/575

For more information, please contact repository@pobox.upenn.edu. 


\title{
Ambipolar transport in solution-deposited pentacene transistors enhanced by molecular engineering of device contacts
}

\author{
Abstract \\ We report ambipolar transport in bottom gold contact, pentacene field-effect transistors (FETs) fabricated \\ by spin-coating and thermally converting its precursor on a benzocyclobutene/SiO 2 gate dielectric with \\ chemically modified source and drain electrodes. A wide range of aliphatic and aromatic self-assembled \\ thiolate monolayers were used to derivatize the electrodes and all enhanced electron and hole currents, \\ yet did not affect the observable thin film morphology. Hole and electron mobilities of $0.1-0.5$ and \\ $0.05-0.1 \mathrm{~cm}^{2} / \mathrm{V}$ s are achieved, though the threshold for electron transport was $>80 \mathrm{~V}$. These ambipolar \\ FETs are used to demonstrate inverters with gains of up to 94 . \\ Disciplines \\ Electrical and Computer Engineering | Engineering

\section{Comments} \\ Suggested Citation: \\ Saudari, S.R., Frail, P.R., Kagan, C.R. (2009). "Ambipolar transport in solution-deposited pentacene \\ transistors enhanced by molecular engineering of device contacts." Applied Physics Letters. 95, 023301. \\ (C) 2009 American Institute of Physics. This article may be downloaded for personal use only. Any other \\ use requires prior permission of the author and the American Institute of Physics. \\ The following article appeared in Applied Physics Letters and may be found at http://dx.doi.org/10.1063/ \\ 1.3177007
}




\title{
Ambipolar transport in solution-deposited pentacene transistors enhanced by molecular engineering of device contacts
}

\author{
Sangameshwar Rao Saudari, Paul R. Frail, and Cherie R. Kagan ${ }^{\text {a) }}$ \\ University of Pennsylvania, Philadelphia, Pennsylvania 19104, USA
}

(Received 3 March 2009; accepted 23 June 2009; published online 13 July 2009)

\begin{abstract}
We report ambipolar transport in bottom gold contact, pentacene field-effect transistors (FETs) fabricated by spin-coating and thermally converting its precursor on a benzocyclobutene $/ \mathrm{SiO}_{2}$ gate dielectric with chemically modified source and drain electrodes. A wide range of aliphatic and aromatic self-assembled thiolate monolayers were used to derivatize the electrodes and all enhanced electron and hole currents, yet did not affect the observable thin film morphology. Hole and electron mobilities of $0.1-0.5$ and $0.05-0.1 \mathrm{~cm}^{2} / \mathrm{V} \mathrm{s}$ are achieved, though the threshold for electron transport was $>80 \mathrm{~V}$. These ambipolar FETs are used to demonstrate inverters with gains of up to 94. (C) 2009 American Institute of Physics. [DOI: 10.1063/1.3177007]
\end{abstract}

Ambipolar transport in organic semiconductors has attracted considerable attention for numerous applications of organic complementary metal-oxide semiconductor (CMOS)-like devices in low cost and flexible electronics ${ }^{1,2}$ and in light-emitting ${ }^{2-4}$ and photosensing ${ }^{5,6}$ field-effect transistors (FETs). However, organic semiconductors are commonly classified as either $n$-type or $p$-type as different materials have typically shown unipolar behavior in FETs with, for example, either exclusively electron transport with mobilities of $\sim 10^{-3}-10^{-1} \mathrm{~cm}^{2} / \mathrm{V} \mathrm{s}$ in arylene diimides $^{7,8}$ or hole transport with mobilities of $\sim 1 \mathrm{~cm}^{2} / \mathrm{V} \mathrm{s}$ in pentacene. ${ }^{9-11}$ Several groups have reported ambipolar organic FETs by fabricating bilayers ${ }^{2,12}$ or blends ${ }^{1,2,4}$ combining an $n$-type and a $p$-type organic semiconductor. Recent reports have established that ambipolar transport is an intrinsic property of organic semiconductors. ${ }^{1,13}$ In many organic thin film semiconductors which were known to be hole conductors, including pentacene, the absence or poor transport of electrons has been attributed to extrinsic factors: (i) high injection barriers for electrons at the metal-semiconductor interface; (ii) electron traps at the dielectric-semiconductor interface; and (iii) electron trap generation upon exposure to different environments.

Fabricating ambipolar FETs having a single organic semiconductor channel material is much simpler compared to bilayers and blends. Single-component ambipolar organic FETs were achieved employing low work function source and drain electrodes or one low work function metal for electron injection and one high work function metal for hole injection. ${ }^{2,14-16}$ The drawbacks of these structures are (i) the poor stability of the low work function electrodes and (ii) two different metals require multiple angled depositions or lithography/masking steps. Ambipolar transport has been reported in low bandgap organic semiconductors using $\mathrm{Au}$ electrodes, but these materials have low mobility. ${ }^{1,16,17}$ Singh et $a l{ }^{18}$ reported ambipolar transport in vacuum deposited pentacene FETs using a polyvinyl alcohol dielectric and $\mathrm{Au}$ electrodes, attributing the ambipolar character to the small grain structure uniquely formed on this dielectric.

In this letter, we report ambipolar transport in solutionprocessed, bottom $\mathrm{Au}$ contact pentacene FETs that is en-

${ }^{a)}$ Electronic mail: kagan@ @eas.upenn.edu. hanced by chemically modifying the source and drain electrodes with self-assembled thiolate monolayers. Previously, self-assembled monolayers (SAMs) were effectively employed in lowering the device contact resistance and improving hole injection in $p$-type small molecule and polymeric FETs fabricated on $\mathrm{SiO}_{2}$ gate dielectrics. ${ }^{19-22}$ Here we bury the $\mathrm{SiO}_{2}$ gate dielectric with benzocyclobutene (BCB), providing a dielectric stack, which eliminates the $\mathrm{SiO}_{2}$ surface sites believed to act as electron traps at the dielectricsemiconductor interface. ${ }^{13}$ We show that then modifying the source and drain electrodes with SAMs, using a wide range of aromatic and aliphatic thiol chemistries, enhances both electron and hole injection forming high-performance ambipolar pentacene FETs. The ambipolar characteristics achieved allow us to demonstrate inverter circuits with high gain.

Pentacene FETs were fabricated in bottom contact geometry [Fig. 1(a)]. $N$-type Si wafers $(\rho<0.01 \Omega$-cm) with $250 \mathrm{~nm}$ thermally grown $\mathrm{SiO}_{2}$ serve as the back gate and part of the gate-dielectric stack of the FETs, respectively. The substrates were transferred into a $\mathrm{N}_{2}$-glove box where all the device fabrication and characterization was performed. The wafers were cleaned by UV-ozone for $20 \mathrm{~min}$. A 1:3 solution of BCB:mesitylene was filtered $[0.2 \mu \mathrm{m}$ polytetrafluoroethylene (PTFE) syringe filter], deposited by spin-coating at $3000 \mathrm{rpm}$ for $30 \mathrm{~s}$, and annealed at $265^{\circ} \mathrm{C}$ for $30 \mathrm{~s}$, providing $\sim 130 \mathrm{~nm}$ BCB layer. The measured capacitance of the $\mathrm{SiO}_{2}(250 \mathrm{~nm}) / \mathrm{BCB}(\sim 130 \mathrm{~nm})$ gate dielectric stack was $7.6( \pm .25) \mathrm{nF} / \mathrm{cm}^{2}$. Au source and drain electrodes $(15-20$ $\mathrm{nm})$ were thermally evaporated through a shadow mask to define channel lengths $(L)$ ranging from 30-200 $\mu \mathrm{m}$ and widths $(W)$ to provide $W / L$ of 15 for each device.

Benzenethiol (1), 4-nitrobenzenethiol 4-aminobenzenethiol (3), 4-sulfanylphenol (4) 2,3,4,5,6pentafluorobezenethiol (5), 1H-pyridine-4-thione (6), naphthalene-2-thiol (7), ethanethiol (8), butanethiol (9), and hexanethiol (10) were purchased from Sigma-Aldrich. Thioketone (11) was synthesized according to literature procedures. $^{20}$ The source and drain electrodes were derivatized from $10 \mathrm{~m} M$ solutions of monolayer forming molecules 1-5 and 7-10 in toluene, $10 \mathrm{~m} M$ of $\mathbf{6}$ in ethanol, and $1 \mathrm{~m} M$ of $\mathbf{1 1}$ in tetrahydrofuran. The substrates were immersed in the molecular monolayer forming solutions for 

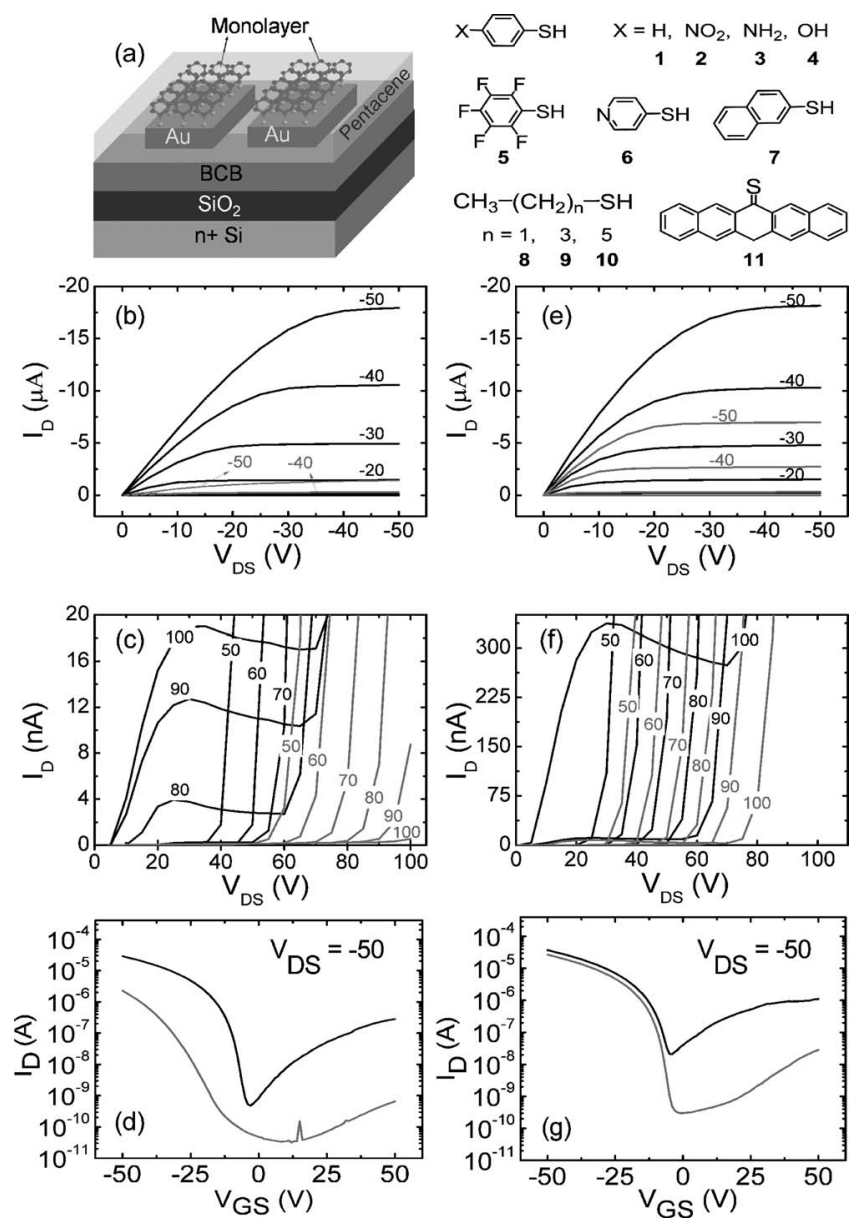

FIG. 1. (a) Device schematic showing surface modification with monolayers of compounds 1-11. Output [(b) and (c)] and transfer characteristics (d) of devices with 1 modified electrode in (b) hole and (c) electron accumulation regimes. Output [(e) and (f)] and transfer characteristics (g) of devices with 8 modified electrodes in (e) hole and (f) electron accumulation regimes. The output and transfer characteristics show thiolate modified devices (black) in comparison with unmodified devices (gray) prepared side-by-side. The channel lengths and widths of all the devices are $200 \mu \mathrm{m}$ and $3 \mathrm{~mm}$, respectively.

15-18 h, rinsed in the fresh parent solvent, and blown dry with $\mathrm{N}_{2}$. A reference set of "unmodified" devices was prepared each time by immersing the devices in the parent solvent with no thiol.

Pentacene was deposited by spin-coating and thermal conversion of its $n$-sulfinylacetamidopentacene precursor. $^{23}$ The precursor was synthesized in-house according to literature procedures, ${ }^{23}$ or provided by IBM or Sigma-Aldrich. The precursor was dissolved in chloroform $(15 \mathrm{mg} / \mathrm{mL})$, filtered through a $0.2 \mu \mathrm{m}$ PTFE syringe filter, and spun at $1500 \mathrm{rpm}$ for $1 \mathrm{~min}$. The precursor thin film was converted at $200{ }^{\circ} \mathrm{C}$ for $1 \mathrm{~min}$ to pentacene.

$I-V$ characteristics of unmodified and modified devices were collected using a Karl Suss PM5 probe station in combination with an Agilent 4156C semiconductor parameter analyzer. Figure 1 shows representative output and transfer characteristics for pentacene FETs with [Figs. 1(b)-1(d)] benzenethiolate $\mathbf{1}$ and [Figs. 1(e)-1(g)] ethanethiolate 8 modified electrodes. $\mathbf{1}$ and $\mathbf{8}$ provide contrasting examples of aromatic and aliphatic monolayers. $I_{\mathrm{D}^{-}} V_{\mathrm{DS}}$ characteristics at high negative $V_{\mathrm{GS}}$ in Figs. 1(b) and 1(e) show hole accumulation, while at high positive $V_{\mathrm{GS}}$ in Figs. 1(c) and 1(f) show electron accumulation characteristic of $p$-channel and

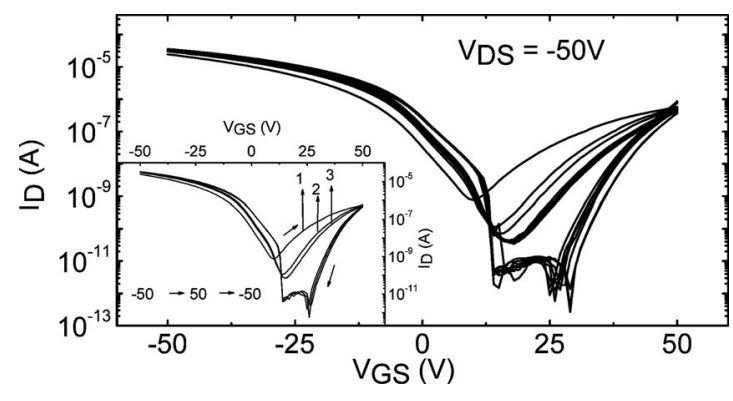

FIG. 2. Hysteresis behavior in the transfer characteristics of a device with 8 modified electrodes. The device was cycled from -50 to +50 to $-50 \mathrm{~V}$ three times (inset), then from +50 to -50 to $+50 \mathrm{~V}$ (three times) and then again -50 to +50 to $-50 \mathrm{~V}$ (three times). All nine accumulated curves shown.

$n$-channel organic FETs. At lower positive $V_{\mathrm{GS}}$ and high $V_{\mathrm{DS}}$, hole accumulation under the drain contact contributes significantly to transport in the channel. $I_{\mathrm{D}^{-}} V_{\mathrm{GS}}[$ Figs. $1(\mathrm{~d})$ and $1(\mathrm{~g})]$ characteristics clearly show ambipolar transport in the solution-deposited pentacene FETs. The drain current in both the hole accumulation and electron accumulation regimes is dramatically improved in devices modified with either $\mathbf{1}$ or $\mathbf{8}$ compared to devices with unmodified electrodes. $I_{\mathrm{D}}-V_{\mathrm{DS}}$ data for unmodified devices, reflective of the $I_{\mathrm{D}}-V_{\mathrm{DS}}$ curves [Figs. 1(d) and 1(g)] show lower hole [Figs. 1(b) and 1(e)] and little to no electron currents [Figs. 1(c) and 1(f)]. The saturation mobility for holes was $0.1-0.5 \mathrm{~cm}^{2} / \mathrm{V} \mathrm{s}$ and for electrons was $0.05-0.1 \mathrm{~cm}^{2} / \mathrm{V} \mathrm{s}$ for $\mathbf{1}$ and $\mathbf{8}$ modified devices. The threshold voltage for hole conduction was -15 to $-30 \mathrm{~V}$ for unmodified electrodes and -1 to $-10 \mathrm{~V}$ for the thiolate modified electrodes. The threshold voltage for electron conduction was 80-90 $\mathrm{V}$ for the thiolate modified electrodes.

All monolayer chemistries 1-11 showed significant improvement in both the hole and electron currents. While there was device-to-device variation in the electron and hole on currents, there was no observable systematic variation in on current with the dipole or electron-withdrawing or electrondonating nature of the compounds that would be anticipated to affect the metal work function. Atomic force microscopy and scanning electron microscopy images show the solutiondeposited precursor route to pentacene forms thin films with the same observable morphologies both in the channel and at the electrode interface for all of the monolayer chemistries as with unmodified electrodes, ${ }^{24}$ suggesting that the thin film structure even at the electrodes is not substantially affected by the surface modification. The only systematic observation was a decrease in on current, with an increase in length of the aliphatic thiols. This same trend in length was not observed in comparing aromatic thiols (1 and 7). Our measurements suggest within our device-to-device variations that the sulfur-gold bond may govern charge injection and act to pin the Fermi level.

Figure 2 shows the hysteresis loop formed by the forward $(-50 \mathrm{~V} \rightarrow+50 \mathrm{~V} \rightarrow-50 \mathrm{~V})$ and reverse $(+50 \mathrm{~V} \rightarrow$ $-50 \mathrm{~V} \rightarrow+50 \mathrm{~V})$ transfer characteristics. The hysteresis depends on applied $V_{\mathrm{GS}}$ and becomes more hysteretic as $V_{\mathrm{GS}}$ is increasingly positive, independent of the sweep direction. The reverse transfer characteristics consistently show greater current modulation and subthreshold slope for both the electron and hole branches and an increased carrier depletion region. The $I_{\mathrm{D}}-V_{\mathrm{GS}}$ characteristics shift in voltage with cycling, Fig. 2 (inset), but become more stable with each cycle, 

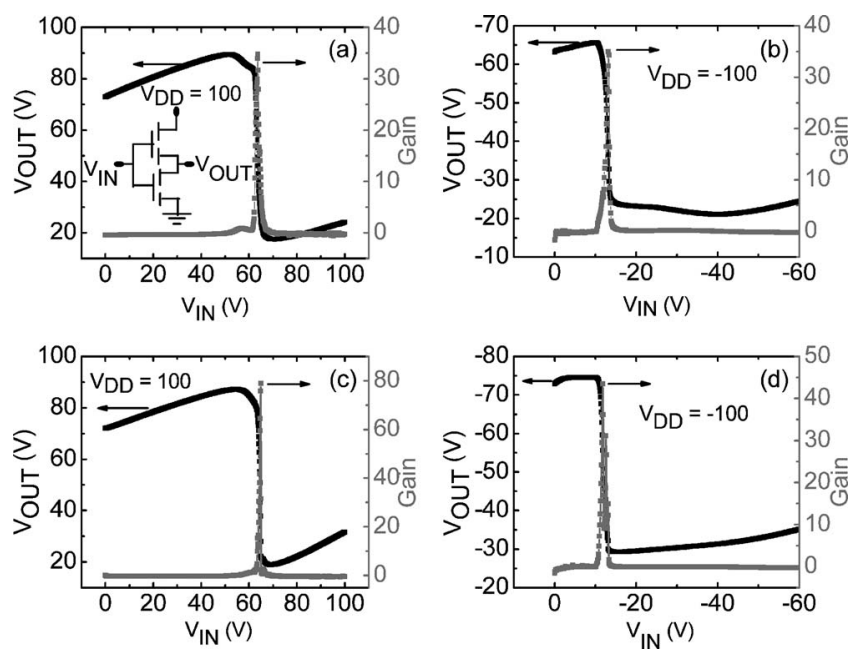

FIG. 3. Transfer characteristics and gain curves of inverters constructed from ambipolar FETs with channel lengths of $200 \mu \mathrm{m}$ and widths of $3 \mathrm{~mm}$. [(a) and (b)] for devices with 1 modified electrode. [(c) and (d)] for devices with $\mathbf{8}$ modified electrodes.

independent of sweep direction. The loop shifts back to its initial state when the device is left idle for a few hours indicating a limited carrier retention time. Little hysteresis is observed when the voltage was swept in only the hole or electron accumulation region. The hysteresis is consistent with electron traps in pentacene or at the pentacene-dielectric interface. ${ }^{25}$ As observed for many ambipolar organic FETs, while the hole current remains stable in air ambient, electron transport is not air stable.

Using the ambipolar FETs achieved by chemically modifying the device electrodes and using the $\mathrm{BCB} / \mathrm{SiO}_{2}$ dielectric stack, bottom contact inverters were fabricated from our solution-processable precursor route to pentacene, employing the $\mathrm{Si}$ wafer as a common gate. Figure 3 shows the inverter circuit and transfer characteristics at [Figs. 3(a) and 3(c)] positive and [Figs. 3(b) and 3(d)] negative supply voltage $\left(V_{\mathrm{DD}}\right)$ for FETs with [Figs. 3(a) and 3(b)] $\mathbf{1}$ and [Figs. $3(\mathrm{c})$ and $3(\mathrm{~d})] \mathbf{8}$ modified electrodes. The inverter shows typical voltage transfer characteristics with low-to-high input voltage driving high-to-low output voltage with gain of $35 / 35$ for positive/negative $V_{\mathrm{DD}}$ for $\mathbf{1}$ modified devices and gain of 79/43 for positive/negative $V_{\mathrm{DD}}$ for $\mathbf{8}$ modified devices. The slope in the transfer characteristics at high and low input voltage arises from the ambipolar nature of the FETs which are never truly off as in conventional CMOS. While Fig. 3 shows inverters using $\mathbf{1}$ and $\mathbf{8}$ modified electrodes, many high gain inverters (with gains of up to 94) were fabricated using the various monolayer chemistries explored. These gains exceed previously published reports of single component inverters. $1,17,18$

Molecular engineering of device contacts is a powerful route to achieving high electron and hole injection efficiency and, in combination with trap-free semiconductor-dielectric interfaces, ambipolar transport in organic FETs from single channel materials and from single metal, environmentally stable, source and drain electrodes. Understanding the significance of the metal-molecule contact chemistry versus the impact of the electronic structure of the entire molecule on the tunability in the energy level alignment of metal-organic interfaces and therefore the contact resistance that governs electron and hole transport in devices is crucial and underway. Ambipolar devices provide a simple route to CMOSlike circuits and higher gain inverters may be achieved by optimizing device geometry to match $n$-channel and $p$-channel characteristics.

We thank Robin Havener for helping to develop processing conditions for BCB. We thank Sigma-Aldrich and Ali Afzali for providing pentacene precursor.

${ }^{1}$ E. J. Meijer, D. M. De Leeuw, S. Setayesh, E. van Veenendaal, B. H. Huisman, P. W. M. Blom, J. C. Hummelen, U. Scherf, and T. M. Klapwijk, Nature Mater. 2, 678 (2003).

${ }^{2}$ J. Zaumseil and H. Sirringhaus, Chem. Rev. (Washington, D.C.) 107, 1296 (2007).

${ }^{3}$ J. Zaumseil, R. H. Friend, and H. Sirringhaus, Nature Mater. 5, 69 (2006).

${ }^{4}$ C. Rost, S. Karg, W. Riess, M. A. Loi, M. Murgia, and M. Muccini, Appl. Phys. Lett. 85, 1613 (2004).

${ }^{5}$ S. Cho, J. Yuen, J. Y. Kim, K. Lee, and A. J. Heeger, Appl. Phys. Lett. 90, 063511 (2007).

${ }^{6}$ T. D. Anthopoulos, Appl. Phys. Lett. 91, 113513 (2007).

${ }^{7}$ M. M. Ling, P. Erk, M. Gomez, M. Koenemann, J. Locklin, and Z. N. Bao, Adv. Mater. (Weinheim, Ger.) 19, 1123 (2007).

${ }^{8}$ B. A. Jones, A. Facchetti, M. R. Wasielewski, and T. J. Marks, Adv. Funct. Mater. 18, 1329 (2008).

${ }^{9}$ Y. Y. Lin, D. J. Gundlach, S. F. Nelson, and T. N. Jackson, IEEE Electron Device Lett. 18, 606 (1997).

${ }^{10}$ M. M. Payne, S. R. Parkin, J. E. Anthony, C. C. Kuo, and T. N. Jackson, J. Am. Chem. Soc. 127, 4986 (2005).

${ }^{11}$ C. R. Kagan, A. Afzali, and T. O. Graham, Appl. Phys. Lett. 86, 193505 (2005)

${ }^{12}$ A. Dodabalapur, H. E. Katz, L. Torsi, and R. C. Haddon, Science 269, 1560 (1995)

${ }^{13}$ L. L. Chua, J. Zaumseil, J. F. Chang, E. C. W. Ou, P. K. H. Ho, H. Sirringhaus, and R. H. Friend, Nature (London) 434, 194 (2005).

${ }^{14}$ T. Yasuda, T. Goto, K. Fujita, and T. Tsutsui, Appl. Phys. Lett. 85, 2098 (2004).

${ }^{15}$ R. Schmechel, M. Ahles, and H. von Seggern, J. Appl. Phys. 98, 084511 (2005).

${ }^{16}$ S. Noro, T. Takenobu, Y. Iwasa, H. C. Chang, S. Kitagawa, T. Akutagawa and T. Nakamura, Adv. Mater. (Weinheim, Ger.) 20, 3399 (2008).

${ }^{17}$ T. D. Anthopoulos, S. Setayesh, E. Smits, M. Colle, E. Cantatore, B. de Boer, P. W. M. Blom, and D. M. de Leeuw, Adv. Mater. (Weinheim, Ger.) 18, 1900 (2006).

${ }^{18}$ T. B. Singh, P. Senkarabacak, N. S. Sariciftci, A. Tanda, C. Lackner, R. Hagelauer, and G. Horowitz, Appl. Phys. Lett. 89, 033512 (2006).

${ }^{19}$ D. J. Gundlach, L. L. Jia, and T. N. Jackson, IEEE Electron Device Lett. 22, 571 (2001).

${ }^{20}$ G. S. Tulevski, Q. Miao, A. Afzali, T. O. Graham, C. R. Kagan, and C. Nuckolls, J. Am. Chem. Soc. 128, 1788 (2006).

${ }^{21}$ C. Bock, D. V. Pham, U. Kunze, D. Kafer, G. Witte, and C. Woll, J. Appl. Phys. 100, 114517 (2006).

${ }^{22}$ B. H. Hamadani, D. A. Corley, J. W. Ciszek, J. M. Tour, and D. Natelson, Nano Lett. 6, 1303 (2006).

${ }^{23}$ A. Afzali, C. D. Dimitrakopoulos, and T. L. Breen, J. Am. Chem. Soc. 124, 8812 (2002).

${ }^{24} \mathrm{See}$ EPAPS supplementary material at http://dx.doi.org/10.1063/ 1.3177007 for AFM and SEM images.

${ }^{25}$ G. Gu and M. G. Kane, Appl. Phys. Lett. 92, 053305 (2008). 